\title{
THE NOETHER-LEFSCHETZ THEOREM VIA VANISHING OF COHERENT COHOMOLOGY
}

\author{
G. V. RAVINDRA
}

\begin{abstract}
We prove that for a generic hypersurface in $\mathbb{P}^{2 n+1}$ of degree at least $2+2 / n$, the $n$-th Picard number is one. The proof is algebraic in nature and follows from certain coherent cohomology vanishing.
\end{abstract}

\section{INTRODUCTION}

Let $X$ be a smooth projective variety and $\mathrm{CH}^{m}(X)$ denote the Chow group of codimension $m$ cycles modulo rational equivalence. There is a cycle class map $\mathrm{CH}^{m}(X) \rightarrow \mathrm{H}^{m, m}(X, \mathbb{Z}):=\mathrm{H}^{2 m}(X, \mathbb{Z}) \cap \mathrm{H}^{m}\left(X, \Omega_{X}^{m}\right)$. The image of this map is called the $m$-th Picard group and the rank of this group is called the $m$-th Picard number and is denoted by $P_{m}(X)$.

The main result in this paper is the following.

Theorem 1 (Noether-Lefschetz theorem). Let $X \subset \mathbb{P}^{2 n+1}$ be a generic smooth hypersurface of degree $d$ at least $2+2 / n$. Then the $n$-th Picard number of $X, P_{n}(X)=1$

This theorem appears as Theorem (13.22) in [5] with the hypothesis $\mathrm{H}^{n, n}(X) \neq \mathrm{H}^{2 n}(X, \mathbb{C})$ when $X$ is a complete intersection. In case $X$ is a hypersurface, this is equivalent to the hypothesis in the above theorem(see 13.23 op . cit.). Our methods can easily be extended to complete intersections, but we restrict our attention to hypersurfaces to keep the presentation as simple as possible.

It is easy to see that the theorem above follows from the Noether-Lefschetz theorem for Hodge classes (see [2]) which states that under the hypothesis of the theorem above $\mathrm{H}^{n, n}(X, \mathbb{Q}) \cong \mathbb{Q}$. Proofs of this stronger version of the Noether-Lefschetz theorem found in op. cit. as well as elsewhere in the literature, use the formalism of Lefschetz pencils and the action of the monodromy group on certain Hodge/coniveau filtrations. Our proof of the weaker Noether-Lefschetz theorem is purely algebraic and follows from the vanishing of cohomologies of certain coherent sheaves/vector bundles and is closer in spirit to Grothendieck's proof of the Grothendieck-Lefschetz theorem.

The outline of the proof is as follows: In the style of [1], we first prove an infinitesimal Noether-Lefschetz theorem (section 3.1). This is established

Key words and phrases. Noether-Lefschetz, algebraic cycles, Picard number. 
using purely coherent cohomology vanishing. We then show that this implies that the geometric generic fibre of the universal family of hypersurfaces has middle Picard number one and then use this to prove the global NoetherLefschetz theorem.

The above theorem is sharp; for $n \geq 1$, any even dimensional quadric hypersurface has two generators (given by the classes of the linear subspaces of maximal dimension) in $\mathrm{CH}^{n}(X)$ which are not homologically equivalent. Moreover when $n=1$, one can recover the "classical" Noether-Lefschetz theorem for surfaces $(\operatorname{Pic}(X) \cong \mathbb{Z}$ when $X$ is generic of degree at least 4). This has been done by N.Mohan Kumar and V.Srinivas in [6] and the method of proof presented here is due to them.

1.1. Conventions. We work over the field of complex numbers though our results are valid over any algebraically closed field of characteristic zero. By a generic point of a variety, we mean a point outside a countable union of proper closed subvarieties and by a general point of a variety, we shall mean a point in a Zariski open set.

\section{Cohomology computations}

2.1. Preliminaries. The computations in this section first appeared in [7], but we include them here for the sake of completeness.

Let $k$ be a field of characteristic zero, $\mathbb{P}:=\mathbb{P}_{k}^{m+1}$ and $W=\mathrm{H}^{0}\left(\mathbb{P}, \mathcal{O}_{\mathbb{P}}(d)\right)$. Let $S:=\mathbb{P}\left(W^{*}\right)$ denote the parameter space of all degree $d$ hypersurfaces in $\mathbb{P}$. One has a short exact sequence

$$
0 \rightarrow \mathcal{V} \rightarrow \mathrm{H}^{0}\left(\mathbb{P}, \mathcal{O}_{\mathbb{P}}(d)\right) \otimes \mathcal{O}_{\mathbb{P}} \rightarrow \mathcal{O}_{\mathbb{P}}(d) \rightarrow 0
$$

where $\mathcal{V}$ is the kernel of the evaluation map $W \otimes \mathcal{O}_{\mathbb{P}} \rightarrow \mathcal{O}_{\mathbb{P}}(d)$. It is not hard to see that $\mathcal{X}:=\mathbb{P}\left(\mathcal{V}^{*}\right) \rightarrow S$ is the universal family of all degree $d$ hypersurfaces. Let $X \subset \mathbb{P}$ be a smooth degree $d$ hypersurface corresponding to a closed point $s \in S$. Let $V:=T_{S, s}^{*}$, the dual of the Zariski tangent space at the point $s \in S$. If $A:=\mathcal{O}_{S, s} / \mathfrak{m}_{s}^{2}=k \oplus V^{*}$, we will denote by $X_{\epsilon}$, the universal hypersurface over $\operatorname{Spec} A$. It is easy to see that $\Omega_{A}^{1} \otimes k \cong V^{*}$.

Let $\mathbb{P}_{A}:=\mathbb{P} \times \operatorname{Spec} A$ and $p: \mathbb{P}_{A} \rightarrow \mathbb{P}, q: \mathbb{P}_{A} \rightarrow$ Spec $A$ denote the two projections. Consider the cotangent sheaf sequence for the inclusion $\iota_{\epsilon}: X_{\epsilon} \hookrightarrow \mathbb{P}_{A}$,

$$
\mathcal{O}_{X_{\epsilon}}(-d) \rightarrow \Omega_{\mathbb{P}_{A}}^{1} \otimes \mathcal{O}_{X_{\epsilon}}=p^{*} \Omega_{\mathbb{P}}^{1} \otimes \mathcal{O}_{X_{\epsilon}} \oplus q^{*} \Omega_{A}^{1} \otimes \mathcal{O}_{X_{\epsilon}} \rightarrow \Omega_{X_{\epsilon}}^{1} \rightarrow 0 .
$$

On restricting this sequence to $X$, we have

(1) $0 \rightarrow \mathcal{O}_{X}(-d) \stackrel{(\alpha, \beta)}{\longrightarrow} \Omega_{\mathbb{P}}^{1} \otimes \mathcal{O}_{X} \oplus V^{*} \otimes \mathcal{O}_{X} \stackrel{(\gamma, \delta)}{\longrightarrow} \Omega_{X_{\epsilon}}^{1} \otimes \mathcal{O}_{X} \rightarrow 0$

Let $F$ denote the polynomial defining $X_{\epsilon} \subset \mathbb{P}_{A}$. On taking cohomology of the sequence $0 \rightarrow \mathcal{O}_{\mathbb{P}} \rightarrow \mathcal{O}_{\mathbb{P}}(d) \rightarrow \mathcal{O}_{X}(d) \rightarrow 0$, we get $0 \rightarrow k \rightarrow W \rightarrow$ $V \rightarrow 0$. Choose a splitting $\theta: V \rightarrow W$, so that $\theta \in W \otimes V^{*}$. Since $W \otimes A=W \oplus W \otimes V^{*}$, we have $F=(f, \theta) \in W \otimes A=\mathrm{H}^{0}\left(\mathbb{P}_{A}, \mathcal{O}_{\mathbb{P}_{A}}(d)\right)$ where $f$ is the polynomial defining $X$. The map $d F$ is then given by $(\tilde{\alpha}, \tilde{\beta})$. It 
is easy to see that $\tilde{\alpha}_{\left.\right|_{X}}=\alpha=d f$. By local computations, we can verify that $\tilde{\beta}_{\left.\right|_{X}}=\beta: \mathcal{O}_{X}(-d) \rightarrow V^{*} \otimes \mathcal{O}_{X}$ is dual to the evaluation map. Notice that the injectivity of $\delta$ follows from the injectivity of $\alpha$. We can also identify $\delta$ with the natural map obtained as follows. We have an exact sequence $q^{*} \Omega_{A}^{1} \rightarrow \Omega_{X_{\epsilon}}^{1} \rightarrow \Omega_{X_{\epsilon} / A}^{1} \rightarrow 0$, which is the relative cotangent sheaf sequence of the family $q: X_{\epsilon} \rightarrow \operatorname{Spec} A$. On restricting this sequence to $X$, we get

$$
0 \rightarrow \Omega_{A}^{1} \otimes \mathcal{O}_{X} \stackrel{\delta}{\rightarrow} \Omega_{X_{\epsilon}}^{1} \otimes \mathcal{O}_{X} \rightarrow \Omega_{X_{\epsilon} / A}^{1} \otimes \mathcal{O}_{X}=\Omega_{X}^{1} \rightarrow 0
$$

The exactness of sequence (1) implies that $\gamma \alpha=-\delta \beta$ and as a consequence, we have the following

Lemma 1 (Mohan Kumar-Srinivas, [6]). There is a commutative diagram:

$$
\begin{aligned}
& 0 \rightarrow \mathcal{O}_{X}(-d) \stackrel{\alpha}{\rightarrow} \Omega_{\mathbb{P}}^{1} \otimes \mathcal{O}_{X} \rightarrow \Omega_{X}^{1} \rightarrow 0 \\
& \downarrow-\beta \quad \downarrow \gamma \\
& 0 \rightarrow V^{*} \otimes \mathcal{O}_{X} \stackrel{\delta}{\rightarrow} \Omega_{X_{\epsilon}}^{1} \otimes \mathcal{O}_{X} \rightarrow \Omega_{X}^{1} \rightarrow 0
\end{aligned}
$$

where the two rows come from the inclusions $X \subset \mathbb{P}$ and $X \subset X_{\epsilon}$ and the map $\beta$ is the natural map.

Taking the $\ell$-th exterior power, we get a commutative diagram

$$
\begin{aligned}
& 0 \rightarrow \Omega_{X}^{\ell-1}(-d) \rightarrow \Omega_{\mathbb{P}}^{\ell} \otimes \mathcal{O}_{X} \rightarrow \Omega_{X}^{\ell} \rightarrow 0 \\
& 0 \rightarrow \Omega(\ell) \quad \rightarrow \Omega_{X_{\epsilon}}^{\ell} \otimes \mathcal{O}_{X} \rightarrow \Omega_{X}^{\ell} \rightarrow 0
\end{aligned}
$$

where $\Omega(\ell)$ (see [4], $5.16(\mathrm{~d})$, page 126) comes equipped with a decreasing filtration $\mathrm{F}^{*} \Omega(\ell)$ satisfying the properties $\mathrm{F}^{1}(\Omega(\ell))=\Omega(\ell), \mathrm{F}^{\ell+1}(\Omega(\ell))=0$ and $\operatorname{gr}_{\mathrm{F}}^{j} \Omega(\ell):=\mathrm{F}^{j}(\Omega(\ell)) / \mathrm{F}^{j+1}(\Omega(\ell))=\Lambda^{j} V^{*} \otimes \Omega_{X}^{\ell-j}$ for $j \geq 1$.

Lemma 2. For a smooth hypersurface $X \subset \mathbb{P}^{2 n+1}, \mathrm{H}^{n}(X, \Omega(n))=0$.

Proof. The proof follows by analysing the sequences

$$
0 \rightarrow \mathrm{F}^{j+1}(\Omega(n)) \rightarrow \mathrm{F}^{j}(\Omega(n)) \rightarrow \operatorname{gr}_{\mathrm{F}}^{j} \Omega=\Lambda^{j} V^{*} \otimes \Omega_{X}^{n-j} \rightarrow 0
$$

For $j \geq 1, \mathrm{H}^{n}\left(X, \Omega_{X}^{n-j}\right)=0$. Thus on taking cohomology, we have a surjection

$$
\mathrm{H}^{n}\left(X, \mathrm{~F}^{j+1}(\Omega(n))\right) \rightarrow \mathrm{H}^{n}\left(X, \mathrm{~F}^{j}(\Omega(n))\right) \forall j \geq 1
$$

Since $\mathrm{H}^{n}\left(X, \mathrm{~F}^{n} \Omega(n)\right)=\mathrm{H}^{n}\left(X, \Lambda^{n} V^{*} \otimes \mathcal{O}_{X}\right)=0$, we are done.

Lemma 3. Let $X \subset \mathbb{P}^{2 n+1}$ be a smooth hypersurface. Then there are isomorphisms

$$
\begin{aligned}
& \mathrm{H}^{n+1}\left(X, \Omega_{X}^{n-1}(-d)\right) \cong \mathrm{H}^{2 n}\left(X, \mathcal{O}_{X}(-n d)\right) \\
& \mathrm{H}^{n+1}\left(X, \Omega_{X}^{n-1}\right) \cong \mathrm{H}^{2 n}\left(X, \mathcal{O}_{X}((-n+1) d)\right.
\end{aligned}
$$


Proof. We consider the exterior powers of the cotangent bundle sequence for the inclusion $X \subset \mathbb{P}$

$$
0 \rightarrow \Omega_{X}^{\ell-1}(-d) \rightarrow \Omega_{\mathbb{P}}^{\ell} \otimes \mathcal{O}_{X} \rightarrow \Omega_{X}^{\ell} \rightarrow 0
$$

together with the sequences

$$
0 \rightarrow \Omega_{\mathbb{P}}^{\ell}(-d) \rightarrow \Omega_{\mathbb{P}}^{\ell} \rightarrow \Omega_{\mathbb{P}}^{\ell} \otimes \mathcal{O}_{X} \rightarrow 0
$$

Using the fact that $\mathrm{H}^{i}\left(\mathbb{P}, \Omega_{\mathbb{P}}^{j}(*)\right)=0$ for $i \neq j$, we get isomorphisms

$$
\mathrm{H}^{n+\ell}\left(X, \Omega_{X}^{n-\ell}(-t)\right) \cong \mathrm{H}^{n+\ell+1}\left(X, \Omega_{X}^{n-\ell-1}(-t+d)\right) \forall 1 \leq \ell<n
$$

This finishes the proof.

Lemma 4. Let $X \subset \mathbb{P}^{2 n+1}$ be a smooth hypersurface. Then the map $\phi$ : $\mathrm{H}^{n+1}\left(X, \Omega_{X}^{n-1}(-d)\right) \rightarrow V^{*} \otimes \mathrm{H}^{n+1}\left(X, \Omega_{X}^{n-1}\right)$ is injective for $d \geq 2+\frac{2}{n}$.

Proof. The map $\phi$ is induced by the composite map of sheaves $\Omega_{X}^{n-1} \otimes$ $\mathcal{O}_{X}(-d)=\Omega_{X}^{n-1}(-d) \rightarrow \Omega(n) \rightarrow \Omega_{X}^{n-1} \otimes V^{*}$ in diagram (3) with $\ell=n$. This map is clearly $1 \otimes-\beta$. Using the identifications in the previous lemma, we can identify the map $\phi$ with the dual of the cup product map

$$
\mathrm{H}^{2 n}\left(X, \mathcal{O}_{X}(-n d)\right) \rightarrow V^{*} \otimes \mathrm{H}^{2 n}\left(X, \mathcal{O}_{X}(-(n-1) d)\right.
$$

The lemma now follows by noting that the cup product map

$$
V \otimes \mathrm{H}^{0}\left(\mathcal{O}_{X}(n d-2 n-2)\right) \rightarrow \mathrm{H}^{0}\left(\mathcal{O}_{X}(n d+d-2 n-2)\right)
$$

is surjective as soon as $\mathrm{H}^{0}\left(\mathcal{O}_{X}(n d-2 n-2)\right) \neq 0$ which happens for all $d \geq 2+\frac{2}{n}$.

Proposition 1. Let $X \subset \mathbb{P}^{2 n+1}$ be a smooth hypersurface. If $d \geq 2+\frac{2}{n}$,

$$
\mathrm{H}^{n}\left(X, \Omega_{\mathbb{P}}^{n} \otimes \mathcal{O}_{X}\right) \cong \mathrm{H}^{n}\left(X, \Omega_{X_{\epsilon}}^{n} \otimes \mathcal{O}_{X}\right)
$$

Proof. Consider the cohomology diagram associated to (3) when $\ell=n$.

$$
\begin{aligned}
& \mathrm{H}^{n}\left(X, \Omega_{X}^{n-1}(-d)\right) \rightarrow \mathrm{H}^{n}\left(X,\left.\Omega_{\mathbb{P}}^{n}\right|_{X}\right) \rightarrow \mathrm{H}^{n}\left(X, \Omega_{X}^{n}\right) \rightarrow \mathrm{H}^{n+1}\left(X, \Omega_{X}^{n-1}(-d)\right) \\
& \mathrm{H}^{n}(X, \Omega(n)) \rightarrow \mathrm{H}^{n}\left(X,\left.\Omega_{X_{\epsilon}}^{n}\right|_{X}\right) \rightarrow \mathrm{H}^{n}\left(X, \Omega_{X}^{n}\right) \rightarrow \mathrm{H}^{n+1}(X, \Omega(n))
\end{aligned}
$$

By Lemma 2, we have $\mathrm{H}^{n}(X, \Omega(n))=0$. Furthermore, by KodairaAkizuki-Nakano theorem (see [3], page 154) $\mathrm{H}^{n}\left(X, \Omega_{X}^{n-1}(-d)\right)=0$. This implies that $\mathrm{H}^{n}\left(X, \Omega_{\mathbb{P}}^{n} \otimes \mathcal{O}_{X}\right) \rightarrow \mathrm{H}^{n}\left(X, \Omega_{X_{\epsilon}}^{n} \otimes \mathcal{O}_{X}\right)$ is injective. To prove surjectivity, it is enough to prove that the right most vertical arrow i.e. the map $\mathrm{H}^{n+1}\left(X, \Omega_{X}^{n-1}(-d)\right) \rightarrow \mathrm{H}^{n+1}(X, \Omega(n))$ is injective. By Lemma 4, the composite map $\phi$

$$
\mathrm{H}^{n+1}\left(X, \Omega_{X}^{n-1}(-d)\right) \rightarrow \mathrm{H}^{n+1}(X, \Omega(n)) \rightarrow V^{*} \otimes \mathrm{H}^{n+1}\left(X, \Omega_{X}^{n-1}\right)
$$

is injective. Thus we are done. 


\section{The Noether-Lefschetz theorem}

3.1. The infinitesimal Noether-Lefschetz theorem. Let $X \subset \mathbb{P}^{2 n+1}$ be a smooth general hypersurface of degree at least $2+2 / n$.

Lemma 5. There exists an exact sequence

$$
0 \rightarrow \mathrm{H}^{n}\left(X,\left.\Omega_{X_{\epsilon}}^{n}\right|_{X}\right) \rightarrow \mathrm{H}^{n}\left(X, \Omega_{X}^{n}\right) \stackrel{\kappa}{\rightarrow} V^{*} \otimes \mathrm{H}^{n+1}\left(X, \Omega_{X}^{n-1}\right) .
$$

Proof. The bottom horizontal sequence in diagram (5) together with the fact that $\mathrm{H}^{n}(X, \Omega(n))=0$, yields an exact sequence

$$
0 \rightarrow \mathrm{H}^{n}\left(X,\left.\Omega_{X_{\epsilon}}^{n}\right|_{X}\right) \rightarrow \mathrm{H}^{n}\left(X, \Omega_{X}^{n}\right) \rightarrow \mathrm{H}^{n+1}(X, \Omega(n)) .
$$

From sequence (4), one can easily see that the map $\mathrm{H}^{n+1}(X, \Omega(n)) \rightarrow V^{*} \otimes$ $\mathrm{H}^{n+1}\left(X, \Omega_{X}^{n-1}\right)$ is injective. The map $\kappa$ in the statement of the lemma is just the composite map $\mathrm{H}^{n}\left(X, \Omega_{X}^{n}\right) \rightarrow \mathrm{H}^{n+1}(X, \Omega(n)) \rightarrow V^{*} \otimes \mathrm{H}^{n+1}\left(X, \Omega_{X}^{n-1}\right)$.

Remark 1. It is standard that the map $\kappa$ is the Kodaira-Spencer map. Thus $\mathrm{H}^{n}\left(X, \Omega_{X_{\epsilon} \mid X}^{n}\right)$ can be identified with the subspace of cohomology classes of type $(n, n)$ in $X$ which deform infinitesimally i.e. they remain of type $(n, n)$ under infinitesimal deformations.

Using the isomorphisms

$$
\mathrm{H}^{2 n}(\mathbb{P}, \mathbb{C}) \cong \mathrm{H}^{n}\left(\mathbb{P}, \Omega_{\mathbb{P}}^{n}\right) \cong \mathrm{H}^{n}\left(X,\left.\Omega_{\mathbb{P}}^{n}\right|_{X}\right) \cong \mathrm{H}^{n}\left(X,\left.\Omega_{X_{\epsilon}}^{n}\right|_{X}\right)
$$

one sees that such a class actually lifts to a cohomology class in the ambient projective space.

Lemma 6. Let $Z$ be a homologically non-trivial cycle in $X$. If $Z$ deforms as an algebraic cycle infinitesimally, then $Z$ is homologically equivalent to a multiple of a codimension n linear section $H_{X}^{n}$ where $H_{X}:=H \cap X, H \subset \mathbb{P}$ is a hyperplane section.

Proof. Let $\eta$ denote the cohomology class of $Z$ in $\mathrm{H}^{n}\left(X, \Omega_{X}^{n}\right)$. Since $Z$ deforms infinitesimally, $\eta \in \mathrm{H}^{n}\left(X, \Omega_{X_{\epsilon}}^{n} \mid X\right)$. From the discussion preceding the statement of the lemma, it is clear that $\eta$ lifts to a class in $\mathrm{H}^{n}\left(\mathbb{P}, \Omega_{\mathbb{P}}^{n}\right)$. Furthermore, since $\eta$ is the class of an algebraic cycle, this implies that $\eta$ lifts to a class in $\mathrm{H}^{n}\left(\mathbb{P}, \Omega_{\mathbb{P}}^{n}\right) \cap \mathrm{H}^{2 n}(\mathbb{P}, \mathbb{Q})$. The latter is a one dimensional $\mathbb{Q}$-vector space generated by the class $H^{n}$. This finishes the proof.

3.2. Global Noether-Lefschetz theorem. In this section, we shall consider the universal family $\mathcal{X} \rightarrow S$ defined over $k=\overline{\mathbb{Q}}$.

Proposition 2. For a smooth hypersurface $X$ in $\mathbb{P}^{2 n+1}$ of degree at least 3 , let $X_{\bar{\eta}}$ denote the geometric generic fibre. Then $P_{n}\left(X_{\bar{\eta}}\right)=1$.

Proof. Let $h$ be the class of the hyperplane section of $X$ and $h^{n}$ denote the class of a codimension $n$ linear section. Since $X$ and $h$ are algebraic, they are defined by a bunch of polynomials. Let $K$ be the field obtained by adjoining the coefficients of all these polynomials to $\mathbb{Q}$. Since the number of 
equations are finite, $K$ is finitely generated over $\mathbb{Q}$. Thus $X, h^{n}$ are defined over a finitely generated field $K / \mathbb{Q}$. Let $\bar{K}$ denote the algebraic closure of $K$ and $\bar{K}(\eta)$ denote the rational function field in $h^{0}\left(\mathcal{O}_{\mathbb{P}}(d)\right)-1$ variables over $K$ (i.e., $\bar{K}(\eta)$ is the function field of $S \times \overline{\mathbb{Q}} \bar{K}$ over the field $\bar{K})$ ). Since $\overline{\mathbb{Q}}(\eta) \subset \bar{K}(\eta)$, we have a map $\eta:=\operatorname{Spec} \bar{K}(\eta) \rightarrow \overline{\operatorname{Spec}} \mathbb{Q}(\eta)$. Composing this map with the natural inclusion $\overline{\operatorname{Spec}} \mathbb{Q}(\eta) \hookrightarrow S$, defines a point $\eta \rightarrow S$ which we will call the $\bar{K}$-generic point of $S$. The corresponding $\bar{K}$-geometric generic point of $S$ is $\bar{\eta}:=\operatorname{Spec} \overline{K(\eta)}$.

Let $Z \in \mathrm{CH}^{n}\left(X_{\bar{\eta}}\right)$ be any cycle. Since $\overline{K(\eta)}$ is algebraically closed, the inclusion $Z \hookrightarrow X_{\bar{\eta}}$ is defined over a finite field extension of the function field $\bar{K}(\eta)$. Let $R$ be the ring obtained by "clearing denominators". Then we see that there exists an étale morphism $U=\operatorname{Spec} R \rightarrow S$ such that $Z$ "spreads" out to a codimension $n$ cycle $\mathcal{Z}$ on $\mathcal{X}_{U}$. By shrinking $U$ further, we may assume that (i) the image of $U$ in $S$ is an open set which does not meet the discriminant locus of the linear system $\mathrm{H}^{0}\left(\mathcal{O}_{\mathbb{P}}(d)\right)$ in $S$ and (ii) $\mathcal{Z}$ is flat over $U$. Now we restrict $\mathcal{Z}$ to the fibres of $\mathcal{X}_{U} \rightarrow U$. Since the map $U \rightarrow S$ is étale, we note that $\mathcal{O}_{U} / \mathfrak{m}_{u}^{i} \cong \mathcal{O}_{S} / \mathfrak{m}_{s}^{i}$, where $s$ is the image of $u$ under the map $U \rightarrow S$. Thus by the infinitesimal Noether-Lefschetz theorem, we see that the restriction of $\mathcal{Z}$ to the fibres over $u \in U$ (which by definition lifts to the first infinitesimal thickenings) is homologically equivalent to a constant multiple, say $m$, of the class of a linear section in $\mathrm{CH}^{n}\left(X_{u}\right)$. By shrinking $U$ further, we may assume that for $\pi: \mathcal{X}_{U} \rightarrow U, \mathrm{H}^{2 n}\left(\mathcal{X}_{U}, \mathbb{Z}\right) \cong \mathrm{H}^{0}\left(U, \mathrm{R}^{2 n} \pi_{*} \mathbb{Z}\right)$ (for instance if $U$ is affine, then the higher cohomologies $\mathrm{H}^{i}\left(U, \mathrm{R}^{2 n-i} \pi_{*} \mathbb{Z}\right)=$ 0 for $i>0$ and so the only contribution from the Leray spectral sequence is from the zeroeth cohomology $\left.\mathrm{H}^{0}\left(U, \mathrm{R}^{2 n} \pi_{*} \mathbb{Z}\right)\right)$.

Let $\mathcal{C}:=\mathcal{Z}-m \mathcal{H}$ where $\mathcal{H}$ is a hyperplane section of $\mathcal{X}_{U}$. Since the restriction of $\mathcal{C}$ to each fibre is homologically trivial, this implies that the global section defined by $\mathcal{C}$ is zero at every stalk. This implies that the class of $\mathcal{C}$ is itself zero in $\mathrm{H}^{2 n}\left(\mathcal{X}_{U}, \mathbb{Z}\right)$. From this we conclude that the cycle $\mathcal{Z}$ on $\mathcal{X}_{U}$ must be homologically equivalent to a multiple of the class of a linear section and in particular this holds for the cycle $Z$ in $X_{\bar{\eta}}$ as well.

Lemma 7. Let $X$ be a smooth $k$-variety, where $k$ is an algebraically closed field of characteristic zero. Assume that the image $\mathrm{CH}^{n}(X) \rightarrow \mathrm{H}^{n}\left(X, \Omega_{X / k}^{n}\right)$ is generated by $h^{n}$ where $h$ is the class of the hyperplane section. Let $L$ be any finitely generated field extension of $k$ and let $Z_{L} \in \mathrm{CH}^{n}\left(X_{L}\right)$. Then $\operatorname{cl}\left(Z_{L}\right) \in L\left[h^{n}\right] \subset \mathrm{H}^{n}\left(X, \Omega_{X_{L} / L}^{n}\right)$

Proof. Let $V:=\mathrm{H}^{n}\left(X, \Omega_{X / k}^{n}\right)$ and let $h^{n}, \rho_{1}, \cdots, \rho_{r}$ be a basis for $V$ as well as $V_{L}:=V \otimes_{k} L$. Since $L$ is finitely generated over $k$ we may assume that $L=$ $k\left(x_{1}, \cdots, x_{p}\right)(y)$ where the $x_{i}$ 's are algebraically independent over $k$ and $y$ is algebraic over $k\left(x_{1}, \cdots, x_{p}\right)$. By clearing denominators of equations defining $Z_{L}$, we may assume that $Z_{L}$ is defined over the $\operatorname{ring} R:=k\left[x_{1}, \cdots, x_{p}\right](y)$. So $Z_{L}$ is a cycle in $\mathcal{X}:=X \times \operatorname{Spec} R$. By localising at an element of $f \in k\left[x_{1}, \cdots, x_{p}\right]$, we may assume that $R:=k\left[x_{1}, \cdots, x_{p}\right]_{f}(y)$ and that 
$Z_{L} \hookrightarrow \mathcal{X}$ is a relative $n$-cycle over $S=\operatorname{Spec} R$ and that $\mathcal{X} \rightarrow S$ is a smooth $S$-scheme. We have a commutative diagram

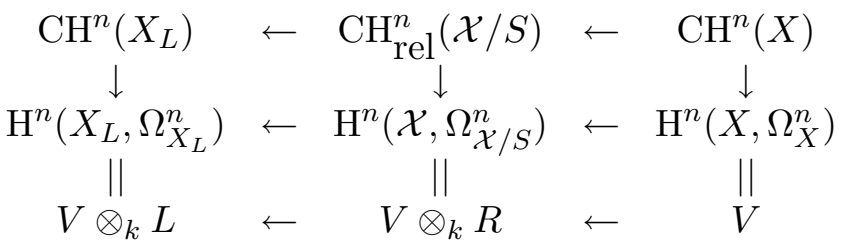

where the top vertical arrows are the cycle class maps, the leftmost left arrows are the restriction maps and the rightmost left arrows are induced by the projection $\mathcal{X} \rightarrow X$.

By construction, $Z_{L}$ is in $\mathrm{CH}^{n}\left(X_{L}\right)$ and $\mathrm{CH}_{\text {rel }}^{n}(\mathcal{X} / S)$. Hence $\operatorname{cl}\left(Z_{L}\right)=$ Image $\left(\operatorname{cl}_{S}\left(Z_{L}\right)\right)=a_{1} h^{m}+a_{2} \rho_{2}+\cdots+a_{r} \rho_{r} \in V \otimes_{k} R$, where $a_{1}, \cdots, a_{r} \in R$. Now for a general point $p=\operatorname{Spec} K \in S$, given by a surjection $R \rightarrow k$, let $Z_{p}$ be the fibre over the point $p$. Then by functoriality of the cycle class map, $\operatorname{cl}\left(Z_{p}\right)=\overline{a_{1}} h^{m}+\overline{a_{2}} \rho_{2}+\cdots+\overline{a_{r}} \rho_{r} \in V$ where $\overline{a_{1}}, \cdots, \overline{a_{r}} \in k$. Here, we use the fact that the composite $X \times p \hookrightarrow \mathcal{X} \rightarrow X$ is the identity. By assumption, $\operatorname{cl}\left(Z_{p}\right)=t h^{n}$ for some $t \in k$. This implies, that for a general point, $\bar{a}_{i}=0$ for $i \geq 2$ which in turn implies that $a_{i}=0$ for $i \geq 2$.

Finally we are in a position to prove theorem 1.

Proof of theorem 1. Let $V$ be the subset of $S_{\mathbb{C}}$ obtained by removing all the divisors of $S_{\mathbb{C}}$, which are defined over $K$. Thus we have removed a countable set of closed subvarieties. If $p \in V$ is a closed point, the map $\operatorname{Spec}(\mathbb{C}(p)) \rightarrow S$ factors through the inclusion of the geometric generic point $\bar{\eta} \hookrightarrow S$. Thus, we have an inclusion of algebraically closed fields $\overline{K(\eta)} \hookrightarrow \mathbb{C}(p) \cong \mathbb{C}$. If $X$ is the fibre over $p \in S_{\mathbb{C}}$, then $X \cong X_{\bar{\eta}} \times \overline{K(\eta)} \mathbb{C}$. In this case, it follows from the above Lemma (see also [8]) that $P_{n}(X)=P_{n}\left(X_{\bar{\eta}}\right)=1$.

\section{AcKnowledgements}

It gives us great pleasure to thank the following people: James Lewis for his insightful comments on the manuscript, C. Maclean for some useful correspondence, N. Mohan Kumar and A. P. Rao for many stimulating discussions on vector bundles in which the idea for proving this result came up, Rao also provided us with a proof of Lemma 7, and helped clarify several aspects of the proof, and finally V. Srinivas for his constant support and encouragement and from whom we first learnt the proof in [6].

\section{REFERENCES}

[1] Carlson, James; Green, Mark; Griffiths, Phillip; Harris, Joe Infinitesimal variations of Hodge structure. I. Compositio Math. 50 (1983), no. 2-3, 109-205.

[2] P. Deligne and N. Katz, Groupes de monodromie en géométrie algébrique. II, Séminaire de Géométrie Algébrique du Bois-Marie 1967-1969 (SGA 7 II). Lecture Notes in Mathematics, Vol. 340. Springer-Verlag, Berlin-New York, 1973. 
[3] Griffiths, Phillip; Harris, Joseph., Principles of algebraic geometry, Wiley Classics Library. John Wiley and Sons, Inc., New York, 1994.

[4] Hartshorne, Robin, Algebraic geometry, Graduate Texts in Mathematics, No. 52. Springer-Verlag, 1977.

[5] Lewis, James D., A survey of the Hodge conjecture, Second edition, CRM Monograph Series, 10. American Mathematical Society, Providence, RI, 1999. xvi+368

[6] N. Mohan Kumar and V. Srinivas, The Noether-Lefschetz theorem, Preprint.

[7] N. Mohan Kumar, A. P. Rao and G. V. Ravindra, Generators for vector bundles on generic hypersurfaces

[8] Terasoma, T., Complete intersections with middle Picard number 1 defined over $\mathbb{Q}$, Math. Z. 189 (1985), no. 2, 289-296.

Department of Mathematics, Washington University in St. Louis, St. Louis, Missouri, 63130

E-mail address: ravindra@math.wustl.edu 